\title{
Experiment GEMMA: Search for the Neutrino Magnetic Moment*
}

\author{
A. Beda, ${ }^{a}$ V. Brudanin, ${ }^{b}$ V. Egorov ${ }^{\dagger},{ }^{b}$ D. Medvedev, ${ }^{b}$ M. Shirchenko, ${ }^{b}$ A. Starostin ${ }^{a}$ \\ ${ }^{a}$ ITEP, Moscow \\ ${ }^{b} J I N R$, Dubna \\ E-mail: eqorovenusun.jinr.ru
}

The result of the 3-year neutrino magnetic moment measurement at the Kalinin Nuclear Power Plant (KNPP) with the GEMMA spectrometer is presented. Antineutrino-electron scattering is investigated. A high-purity germanium detector of $1.5 \mathrm{~kg}$ placed at a distance of $13.9 \mathrm{~m}$ from the centre of the $3 \mathrm{GW}_{\text {th }}$ reactor core is used in the spectrometer. The antineutrino flux is $2.7 \times 10^{13} \bar{v}_{e} / \mathrm{cm}^{2} / \mathrm{s}$. The differential method is used to extract $v$-e electromagnetic scattering events. The scattered electron spectra taken in 5184+6798 and 1853+1021 hours for the reactor $O N$ and $O F F$ periods are compared. The upper limit for the neutrino magnetic moment $\mu_{v}$ was found to be $3.2 \times 10^{-11} \mu_{\mathrm{B}}$ at $90 \% \mathrm{CL}$.

35th International Conference of High Energy Physics - ICHEP2010,

July 22-28, 2010

Paris France

* Supported by the Russian State Corporation ROSATOM and by the Russian Foundation for Basic Research, projects 09-02-00449 and 09-02-12363.

† Speaker. 
The Minimally Extended Standard Model predicts a very small magnetic moment for the massive neutrino $\left(\mu_{v} \sim 10^{-19} \mu_{\mathrm{B}}\right)$ which cannot be observed in an experiment at present. On the other hand, there is a number of extensions of the theory beyond the SM where the Majorana neutrino magnetic moment (NMM) could be at the level of $10^{-(10 \ldots 12)} \mu_{\mathrm{B}}$ irrespective of the neutrino mass, whereas the Dirac NMM could not exceed $10^{-14} \mu_{\mathrm{B}}$ (see, e.g, [四] and references therein). Therefore, observation of an NMM value higher than $10^{-14} \mu_{\mathrm{B}}$ would be evidence for New Physics and, in addition, indicate undoubtedly that the neutrino is a Majorana particle. That is why it is rather important to make laboratory NMM measurements sensitive enough to reach the $\sim 10^{-11} \mu_{\mathrm{B}}$ region. However, the sensitivity of reactor experiments only increased by a factor of three since the Savanna River experiment by Reines' group: from $(2 \ldots 4) \times 10^{-10} \mu_{\mathrm{B}}$ [回] to $(6 \ldots 7) \times 10^{-11} \mu_{\mathrm{B}}$ [目, 田]. Similar limits were obtained for solar neutrinos [[1, 目], but due to oscillations at long distance (as well as matter-enhanced oscillations in the Sun) their flavor composition changes and therefore the solar NMM results could differ from the reactor ones.

A laboratory measurement of the NMM is based on its contribution to the $v-e$ scattering. For nonzero NMM the $v$-e differential cross section is given [] by a sum of the weak interaction cross section $\left(d \sigma_{W} / d T\right)$ and the electromagnetic cross section $\left(d \sigma_{E M} / d T\right)$. At a low recoil electron energy $\left(T \ll E_{V}\right)$ the value of $d \sigma_{W} / d T$ becomes almost constant, while $d \sigma_{E M} / d T$ behaves as $T^{-1}$, so that the lowering of the detector threshold leads to a considerable increase in the NMM effect with respect to the weak unremovable contribution. ${ }^{1}$

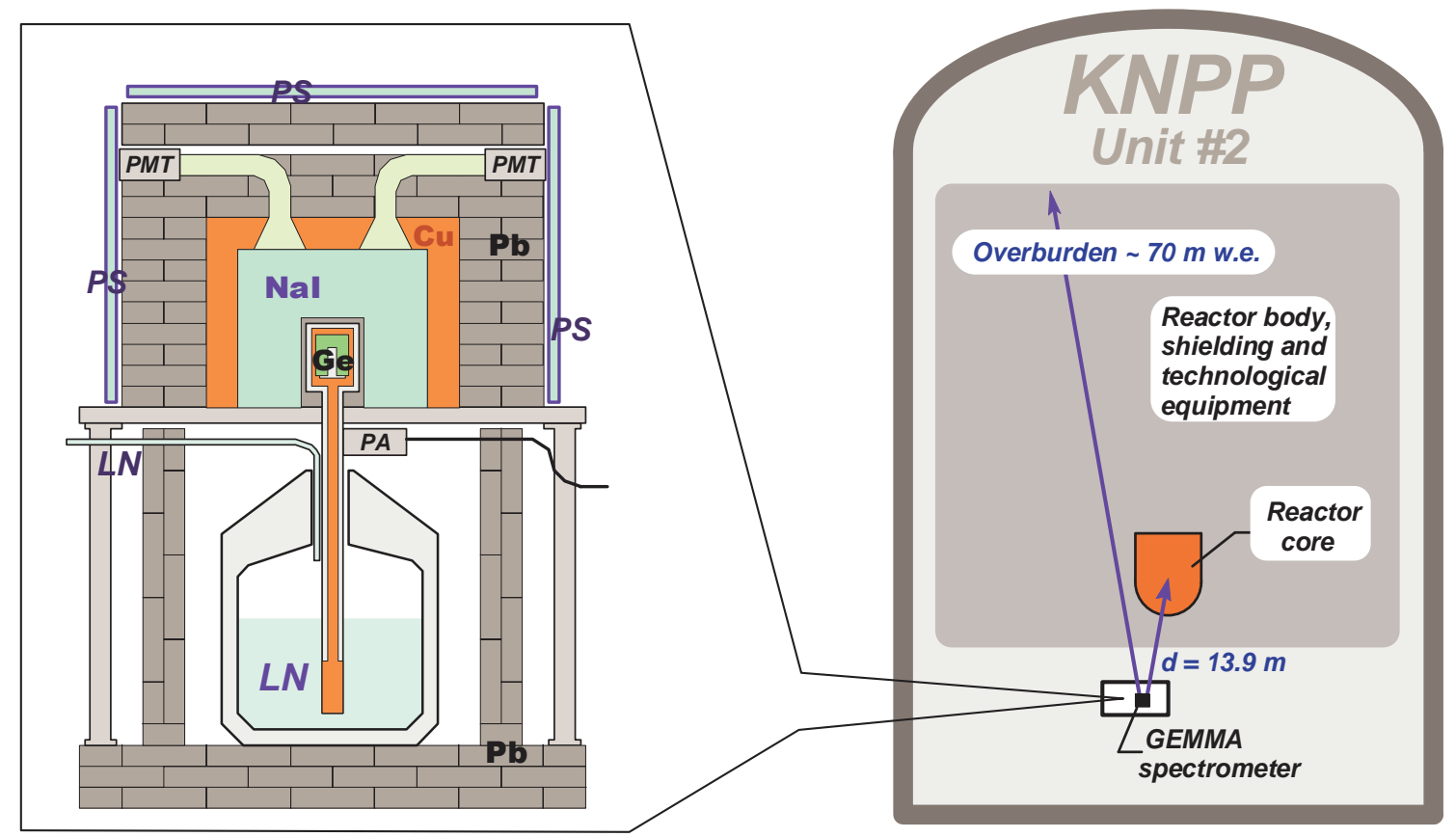

Figure 1: Ge detector inside the active (NaI, PS) and passive $(\mathrm{Cu}, \mathrm{Pb})$ shielding.

To realize this useful feature in our GEMMA spectrometer [䧃], we use a $1.5 \mathrm{~kg}$ HPGe detector with an energy threshold as low as $3 \mathrm{keV}$. The background is suppressed in several steps. First, the

\footnotetext{
${ }^{1}$ According to H. T. Wong et al. [四], the NMM effect could be significantly enhanced by atomic ionization, but the magnitude of such enhancement is questionable [ [8].
} 
detector is placed inside a cup-like NaI crystal with $14 \mathrm{~cm}$ thick walls surrounded with $5 \mathrm{~cm}$ of electrolytic copper and $15 \mathrm{~cm}$ of lead (Fig. W). Active and passive shielding reduces the external $\gamma$ background in the ROI to a level of $\sim 2$ counts $/ \mathrm{keV} / \mathrm{kg} /$ day. Being located just under reactor \#2 of the KNPP (at a distance of $13.9 \mathrm{~m}$ from the reactor core, which corresponds to an antineutrino flux of $2.7 \times 10^{13} \bar{v}_{\mathrm{e}} / \mathrm{cm}^{2} / \mathrm{s}$ ), the detector is well shielded against the hadronic component of cosmic rays by the reactor body and technological equipment (overburden $\simeq 70 \mathrm{~m}$ w.e.). To suppress lowenergy background caused by elastic scattering of secondary neutrons (produced by cosmic muons in the massive $\mathrm{Pb}+\mathrm{Cu}$ shielding), the spectrometer is covered with additional plastic scintillator plates (PS) which generate relatively long $\mu$-veto signals.
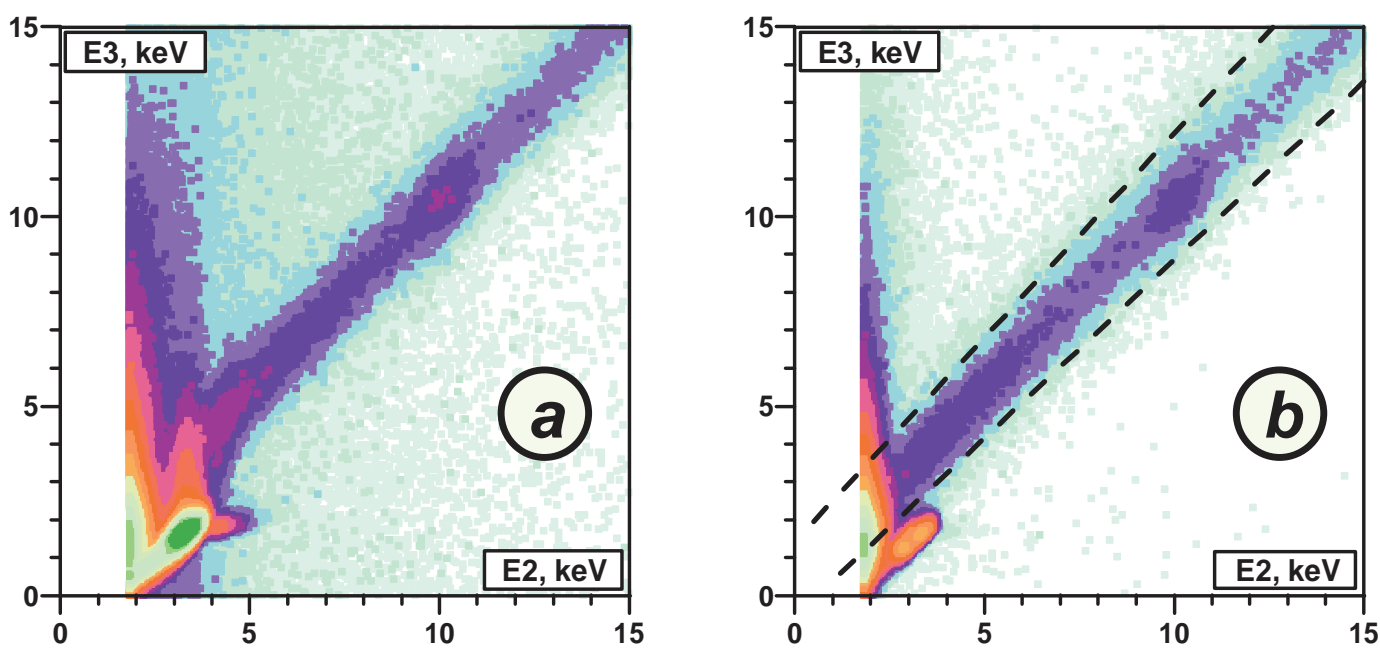

Figure 2: Example of the Fourier analysis made with different shaping-times: ADC-2 and ADC-3 operate with $4 \mu$ s and $12 \mu$ s pulses, respectively. Plot (a) is made before and (b) - after the "audio-frequency" rejection; it is seen that most of the rejected events are non-diagonal. (The color intensity scale is logarithmic.)

Special care is taken to reduce non-physical low-amplitude circuit noise (afterpulses, radio frequency interference, microphonism, etc.). Thus, for example, we reject those events which are separated by a time interval shorter than $80 \mathrm{~ms}$ or equal to $(n \cdot 20.0 \pm 0.1) \mathrm{ms}$. With this "audiofrequency" rejection we suppress the noise caused by mechanical vibrations ("ringing") and the $50 \mathrm{~Hz}$ power-line frequency. In addition, the detector signal is processed by three parallel independent electronic channels with different shaping time $(2,4$ and $12 \mu \mathrm{s})$, which allows a primitive Fourier analysis (Fig. [2) to be performed à posteriori, so artefact signals are discriminated.

In order to get a recoil electron spectrum, we use a differential method comparing the spectra measured during the reactor operation $(\mathrm{ON})$ and shutdown $(\mathrm{OFF})$ periods, the last one being considered as a background. In our previous work [䧃] we presented Phase-I (13 months' measurement including 5184 and 1853 hours of the reactor ON and OFF periods, respectively). Today we can add Phase-II - 19 months from 09.2006 to 05.2008. Unfortunately, for some organizational and technical reasons, there were several interruptions in the measurement. After the preliminary selection, 6798 ON-hours and 1021 OFF-hours of active time were found to be available for analysis.

Fitting the background OFF spectrum in the ROI from $2.9 \mathrm{keV}$ to $55 \mathrm{keV}$ with a parametrized smooth function (Fig. [3]) and comparing the ON spectrum channel by channel with the obtained background curve, we extract their normalized difference $X \equiv \frac{\mathrm{ON}-\mathrm{OFF}-\text { Weak }}{\text { Electromagnetic }}$ which actually repre- 
sents the NMM value squared (in terms of $10^{-11} \mu_{\mathrm{B}}$ ). Averaging over the total ROI and adding the data of the Phase-I, we get at the $90 \% \mathrm{CL}$ the following upper limit: $\mu_{v} \leq 3.2 \times 10^{-11} \mu_{\mathrm{B}}$, which could be an order of magnitude lower if the Atomic Ionization Enhancement [ $[\mathrm{d}]$ really exists (in this case our analysis gives the limit as low as $\mu_{v} \leq 0.5 \times 10^{-11} \mu_{\mathrm{B}}$ ).

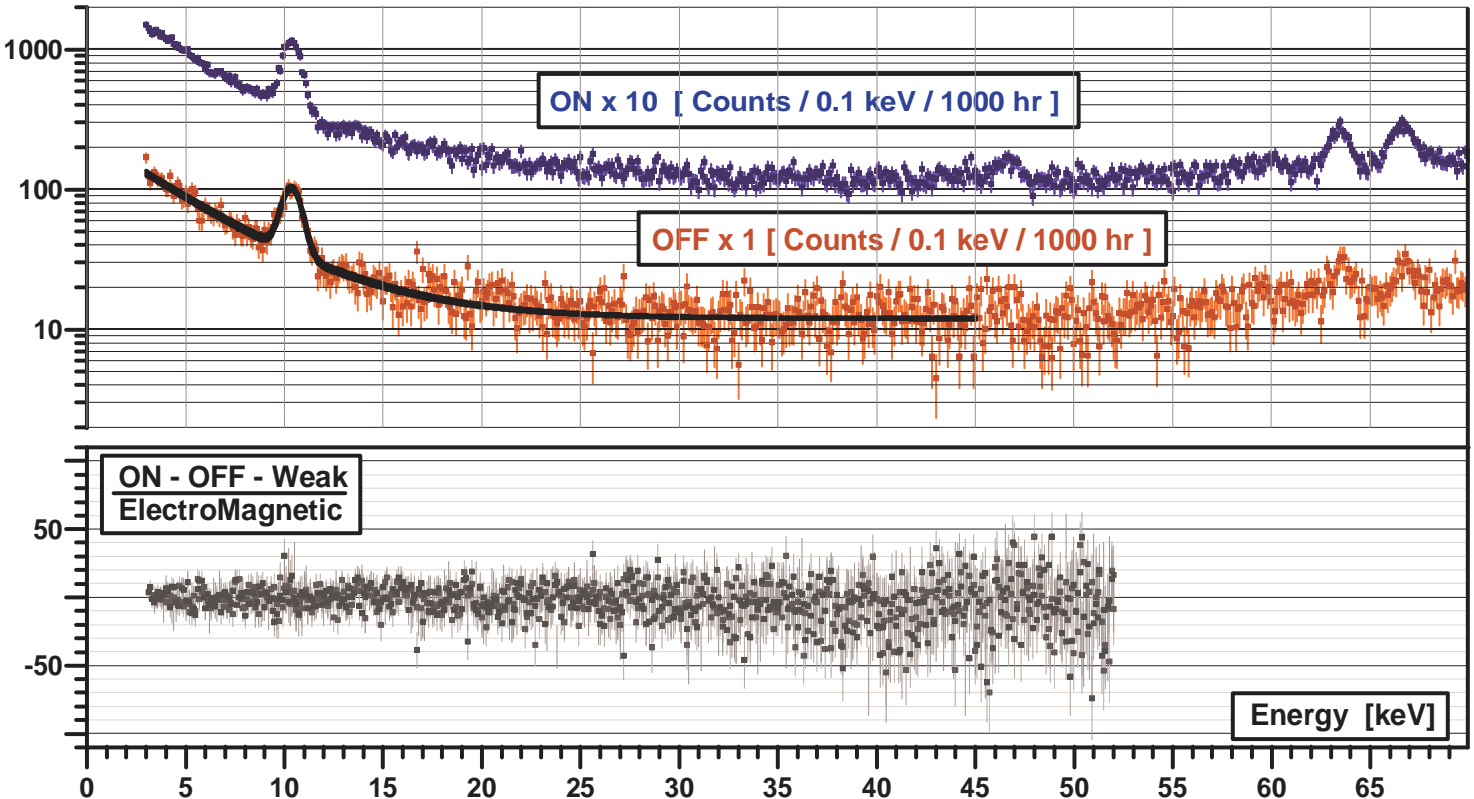

Figure 3: Fragments of the experimental ON and OFF spectra measured in Phase-II, as well as their difference normalized by the electromagnetic cross section. (Only a part of the available statistics is presented.)

\section{References}

[1] C. Giunti and A. Studenikin, Neutrino Electromagnetic Properties, Phys. At. Nucl. 72 (2009) 2089-2125 [hep-ph/0812.3646].

[2] P. Vogel and J. Engel, Neutrino electromagnetic form factors, Phys. Rev. D39 (1989) 3378-3383.

[3] H.T. Wong et al. (TEXONO), Search of neutrino magnetic moments with a high-purity germanium detector at the Kuo-Sheng nuclear power station, Phys. Rev. D75 (2007) 012001 [hep-ex/0605006].

[4] A.G. Beda et al., The first result of the neutrino magnetic moment measurement in the GEMMA experiment, Phys. At. Nucl. 70 (2007) 1873 [hep-ex/ 0705.4576$].$

[5] D.W. Liu et al. (Super-Kamiokande), Limit On the Neutrino Magnetic Moment Using 1496 Days of Super-Kamiokande-i Solar Neutrino Data, Int. J. Mod. Phys. A20 (2005) 3110 [hep-ex/0402015].

[6] C. Arpesella et al. (The Borexino), New results on solar neutrino fluxes from 192 days of Borexino data, Phys. Rev. Lett. 101 (2008) 091302 [astro-ph / 0805.3843 ].

[7] H.T. Wong, H.-B. Li, S.-T. Lin, Enhanced Sensitivities for the Searches of Neutrino Magnetic Moments through Atomic Ionization, Phys. Rev. Lett., 105 (2010) 061801 [hep-ph/1001.2074].

[8] M.B. Voloshin, Neutrino scattering on atomic electrons in searches for neutrino magnetic moment, Phys. Rev. Lett. 105 (2010) 201801 [hep-ph/1008 .2171]. 